\title{
Pregnancy gingivitis and causal inference
}

\section{Abstracted from}

Figuero E, Carrillo-de-Albornoz A, Martín C, Tobías A, Herrera D.

Effect of pregnancy on gingival inflammation in systemically healthy women: a systematic review. J Clin Periodontol 2013; 40: 457-473. doi: 10.1111/jcpe.12053. PubMed PMID: 23557432.

Address for correspondence: Elena Figuero, Departamento de Estomatologia_III.

Facultad de Odontologia Universidad Complutense de Madrid Plaza Ramon y Cajal, S/N.

28040 Madrid, Spain. E-mail: efigueruiz@gmail.com

\section{Question: What is the effect of pregnancy on systemically healthy women in terms of gingival inflammation?}

Data sources The PubMed and Embase databases were searched together with hand searching of the Journal of Periodontology, Journal of Periodontal Research and Journal of Clinical Periodontology. The reference lists of identified articles were also searched.

Study selection Prospective cohort or cross-sectional studies assessing the effect of pregnancy on gingival inflammation evaluated by the gingival index and/or bleeding on probing were included. Study quality was assessed using the Newcastle-Ottawa scale (NOS).

Data extraction and synthesis Study assessment and data extraction were carried out independently by two reviewers, with disputes resolved by a third reviewer. Mean values of primary and secondary outcomes were directly pooled and analysed with weighted mean differences (WMDs) and 95\% confidence intervals (Cls), considering independently each study design (cohort and cross-sectional). Study specific estimates were pooled with both the fixed- and random-effect models.

Results Forty-four articles representing 33 studies (14 cohort and 19 cross-sectional) were included. Meta-analyses revealed a significantly lower $\mathrm{Gl}$ in pregnant women in the first term compared with those in their second or third term of pregnancy; a lower mean GI score in postpartum women compared with women in their second [WMD $=0.143$; $95 \% \mathrm{Cl}(0.031 ; 0.255) ; \mathrm{p}=0.012]$ or third term [WMD $=0.256 ; 95 \%$ $\mathrm{Cl}(0.151 ; 0.360) ; \mathrm{p}<0.001]$ of pregnancy, when considering cohort studies; non-pregnant women had lower mean Gl values than women in their second or third term of pregnancy. Small changes in plaque levels were reported.

Conclusions The results of this systematic review confirm that gingival inflammation is significantly increased throughout pregnancy and when comparing pregnant versus post-partum or non-pregnant women, without a concomitant increase in plaque levels. However, this information should be considered with caution, due to the small number of studies included in the meta-analyses, the low quality of the included studies, differences in study design, absence of a periodontal diagnosis at baseline and performance of periodontal treatment in some cases. No conclusions could be drawn regarding secondary outcomes such as microbiological, immunological and patient-centred data, because no meta-analyses were possible for these factors. Future studies with higher quality should be designed to answer these questions.

\section{Commentary}

According to Wikipedia: 'Old wives' tale is a derogatory epithet used to indicate that a supposed truth is actually a superstition or something untrue, to be ridiculed. The phrase comes with the assumption that a story told by old women could not have credibility, regardless of the particulars of the story. The phrase is used in the context of unvalued women's knowledge...Today, some 'old wives' tales' have proven to be valid.'

One such tale is that women loose a tooth for each child they bear. This, in the US, turns out to be true. ${ }^{1}$ Or perhaps more accurately stated, parity and tooth loss are associated, independent of age and socioeconomic position. A common explanation for this tooth loss is the occurrence of pregnancy gingivitis and periodontitis. ${ }^{2,3}$ Highlighting this relationship is the association of periodontal disease and adverse pregnancy outcomes. ${ }^{4}$

In the current systematic review the authors examined the impact of pregnancy on gingivitis to determine whether there might be a causal relationship. The larger key conceptual series of questions are: does pregnancy cause/lead to/facilitate gingivitis; does gingivitis cause/lead to/facilitate periodontitis; does periodontitis cause/ lead to/facilitate adverse pregnancy outcomes?

The authors identified 14 cohort and 19 cross-sectional studies that met their inclusion criteria. The authors provided extensive tables delineating the materials, methods, gingival/periodontal assessment(s), treatment, outcomes and authors' conclusions of the cohort and cross-sectional studies (Tables 1 and 2). Tables 3-5 provide meta-analytic assessments of gingival index, plaque index and pocket depth respectively. They also analysed the results by trial type (cohort and cross-sectional) and trimester.

Because the cohort studies are better able to predict causality, the following comments focus only on the cohort studies. Gingivitis increased in the second and third trimester compared to the first trimester and women who were not pregnant. Conversely, postpartum women had less gingivitis than women in their second and third trimester. These differences were statistically significant, and they validate the temporal nature of gingivitis during and after pregnancy.

In a larger sense, however, how do these results compare with Bradford Hill's nine elements for demonstrating a causal relationship?

1. Strength of association. The meta-analysis of cohort trials provides a clear demonstration of statistical significance. However, to accomplish this analysis the authors converted trial results 
to weighted mean differences. Weighted mean difference, while statistically sound, provides little information on clinical significance.

2. Consistency. There is consistency among the small trials identified. However, there was also significant heterogeneity among the trials, raising some questions about the strength of association.

3. Specificity. The relationship of smoking and lung cancer was the focus of Bradford Hill's most noted work, and in thinking about the specificity of causal relationships. Pregnancy and gingivitis, while occurring in the same body, similar to smoking, occur in anatomically distinct locations.

4. Temporality. The data clearly indicate that gingivitis increases with the trimester of pregnancy and decreases post-partum.

5. Biological gradient. Similar to temporality, as the mother becomes 'more pregnant' and 'less pregnant' gingivitis increases and decreases, respectively.

6. Plausibility. Biological plausibility 'depends upon the biological knowledge of the day.' There is now a wealth of data demonstrating alteration in hormones, cytokines, inflammatory mediators etc, that change in concert with pregnancy, and that can affect gingival inflammation.

7. Coherence. The data do align with the general knowledge of gingivitis, pregnancy and the effects of hormones on gingival tissue.

8. Experiment. The identified experiments compared women who were not pregnant, who were pregnant and who were post-partum, providing the best available comparisons for experimental design.
9. Analogy. Other studies demonstrate that anti-inflammatory agents reduce gingival inflammation, and that inflammatory cytokines are present during gingival inflammation.

As pointed out by Bradford Hill, none of the nine elements individually or en masse indisputably demonstrate a causal relationship. Rather they assist us in answering the question of whether there is a reasonable explanation for the results we find. By my reckoning, items 1, 2 are a 'maybe', 3 is a 'no', and 4-9 are a 'yes'. Hence my qualitative take home is that there is a causal relationship. However, the strength of this relationship (in other words how great the gingival effect), remains uncertain to me (see \#1, but also \#2 and \#3).

Richard Niederman

Department of Epidemiology and Health Promotion, New York University College of Dentistry, New York, New York, USA

1. Russell SL, Ickovics JR, Yaffee RA. Exploring potential pathways between parity and tooth loss among American women. Am J Public Health 2008; 98: 1263-1270.

2. Loe $\mathrm{H}$, Silness J. Periodontal disease in pregnancy. I. prevalence and severity. Acta Odontol Scand 1963; 21: 533-551.

3. Silness I, Loe H. Periodontal disease in pregnancy. II: correlation between oral hygiene and periodontal condition. Acta Odontol Scand 1964; 22: 121-125.

4. Hill AB. The environment and disease: Association of causation? Proc R Soc Med 1965; 58: $295-300$.

5. Ide M, Papapanou PN. Epidemiology of association between maternal periodontal disease and adverse pregnancy outcomes - systematic review. J Periodontol 2013; 84: S181-194.

Evidence-Based Dentistry (2013) 14, 107-108. doi:10.1038/sj.ebd.6400966 